\title{
Parteien und Kandidatenaufstellung - überzeugende Analyse der Rekrutierung deutscher MdEP
}

\author{
Höhne, Benjamin: Rekrutierung von Abgeordneten des Europäischen Parlaments. Organisation, \\ Akteure und Entscheidungen in Parteien, Verlag Barbara Budrich, Opladen / Berlin / Toronto \\ 2013, 449 Seiten, € 49,90.
}

Die Rekrutierung politischen Personals ist eine zentrale und wichtige Aufgabe von Parteien, da die Kandidaten im Falle ihrer Wahl als Abgeordnete zur politischen Elite eines Gemeinwesens gehören und ganz wesentlich über allgemeinverbindliche Regelungen entscheiden. Die Frage, wie die Auswahl geeigneter Aspiranten durch die Parteien funktioniert, hat deshalb große Bedeutung sowohl für die politikwissenschaftliche Forschung als auch für an praktischen Fragen Interessierte. Davon zeugen nicht zuletzt die jüngeren Diskussionen innerhalb der deutschen Parteien über geeignete Auswahlverfahren beispielsweise von Spitzenkandidaten.

In der vorliegenden Arbeit geht es nun speziell um die Rekrutierung von Kandidaten für die Wahlen zum Europäischen Parlament. Nach wie vor sind die nationalen Parteien bei dieser supranationalen Wahl die Schaltstellen der Vorauswahl für die Listenkandidaten. Wie genau sich die Aufstellung der Landeslisten der CDU in Berlin, Hessen, Nordrhein-Westfalen, Rheinland-Pfalz und Sachsen-Anhalt sowie der CSU in Bayern beziehungsweise der Bundeslisten von SPD, FDP, Bündnis 90/Die Grünen und Die Linke vollzieht und welche Faktoren dabei eine Rolle spielen, ist Gegenstand der von Benjamin Höhne vorgelegten Rekrutierungsanalyse. Der Autor untersucht die Kandidatenauswahl sowohl längsschnittartig als auch gezielt anhand des Rekrutierungsprozesses für die Europawahl des Jahres 2009 mittels qualitativer Interviewdaten wie anhand von durch standardisierte Fragebögen erhobener quantitativer Daten. Das methodische Vorgehen ist dankenswerterweise innerhalb eines mehr als 50-seitigen Anhangs ausführlich dokumentiert.

Das Erkenntnisinteresse beschränkt sich aber keineswegs auf die Deskription, sondern es geht dem Verfasser auch darum, das Agieren der Parteien in ihrem Binnen- und Außenkontext zu erklären. Es soll nämlich untersucht werden, wann es bei der Kandidatenaufstellung zu Beharrung und wann es zu Adaption unter veränderten Umweltzuständen kommt. Die dafür zur Erklärung herangezogenen Variablen sind Kontextbedingungen und innerparteiliche Gegebenheiten. Insgesamt, und daran ist die Arbeit letztlich zu messen, ist es das Ziel, die Parteien- und Parlamentarismusforschung zu bereichern, indem ein weiterer Baustein in das „politikwissenschaftliche Theorie- und Empirie-Gebäude (...) eingepasst (...)“ wird (S. 24).

Benjamin Höhne hält sich nicht lange mit der Darstellung des von ihm überblicksartig wiedergegebenen Forschungsstands und des Untersuchungsgangs auf, sondern geht rasch zu einer gekonnten Skizzierung vorhandener Repräsentationstheorien und einer Beschreibung der Kandidatenaufstellung in unterschiedlichen „Parteiorganisationsmodellen“ über. Auf dieses, als erweiterte Einleitung zu begreifende Kapitel folgt eine detaillierte und sehr kenntnisreiche Übersicht über jene Variablen, die für die Rekrutierung relevant sind, wobei einzelne Subkategorien mit jeweils „zentralen Fragen“ abgeschlossen werden. Dabei räumt der Autor selbst ein, dass sie nicht alle beantwortet werden können. Das Ergebnis der Sammlung und Erläuterung aller wichtigen Kategorien mündet schließlich in einem als Marktmodell bezeichneten Forschungsdesign, in dessen Mittelpunkt als zentrale Akteure auf der einen 
Seite Selektoren und Elektoren, auf der anderen Aspiranten und Interessenten einer Kandidatur stehen (S. 110). Diese agieren innerhalb eines europapolitischen Rahmens, dessen Darstellung (abgesehen von kleinen Nachlässigkeiten, wie etwa der durch das Inkrafttreten des Lissabon-Vertrags abgeschafften Unterscheidung von obligatorischen und nicht-obligatorischen Haushaltsmitteln, S. 117), tadellos gelingt. Neben europapolitischen sind vor allem parteispezifische Kontextvariablen wie Heterogenität und Homogenität, unterschiedliche Strömungen oder der Stellenwert des Föderalismus von Bedeutung. Bei der „Verortung im Parteiensystem“ hätte die ansonsten fehlerfreie Präsentation noch hinzugewonnen, wenn nicht nur das nationale, sondern gerade auch das europapolitische Profil der einzelnen Parteien schärfer akzentuiert worden wäre.

Die Stärke der anschließenden Längsschnittanalyse ist es vor allem, unterschiedliche Typen von Kandidaten herauszuarbeiten, wobei der „Politikaussteiger“ ein seltener werdender Typus ist, während „nationale“ und „europäische Karrieristen“ ebenso wie „flexible Karrieristen " häufiger werden. Durchweg informativ sind außerdem die einwandfrei recherchierten und mit zahlreichen Tabellen und Grafiken versehenen Abschnitte zu Anreizstrukturen, Nachfragesituation und Auswahlkriterien, wie etwa Geschlecht, Alter oder Beruf. Der größte Erkenntniszuwachs geht schließlich von der Querschnittanalyse aus. Hier entfaltet der Autor seine ganze analytische Potenz. Es werden dem Leser nicht nur die Ergebnisse der Befragungen von Aspiranten und Elektoren präsentiert, sondern der Text vermittelt auch tiefgehende Einsichten zu den unterschiedlichen Wahrnehmungen dieser beiden Gruppen hinsichtlich des Anforderungspotentials der Kandidaten. Obendrein gibt es immer wieder durch Fallbeispiele illustrierte Passagen, die die zentrale Rolle von Selektoren verdeutlichen (pars pro toto S. 251 f.) und damit etwa die höchst plausible Unterscheidung von Vorabund Letztentscheidungsarena nachzeichnen. Dieser Abschnitt des Buches liefert eine umfassende Analyse verschiedener Variablen und eine methodische Weiterführung, die ganz besonders durch die Ausarbeitung von jeweils ausführlich hergeleiteten Indizes etwa zu Listenplatz, Wettbewerbssituation oder Abgeordnetendurchsetzung besticht.

Am Ende stellt Benjamin Höhne fest, dass Innen- und Außenwelt der Rekrutierung nicht so gut zusammenpassen, wie sie vielleicht zusammenpassen könnten, so dass er einen dreistufigen Reformvorschlag unterbreitet, der die allgemeinen Partizipationschancen erhöhen und so zu einer besseren Kandidatenauswahl verhelfen könnte. Offen bleibt allerdings, ob für derlei Reform wirklich eine Nachfrage auf Seiten der Wählerschaft existiert und somit diese tatsächlich eine Anpassung an veränderte Umweltzustände darstellte oder ,bloß` demokratietheoretisch wünschenswert wäre.

Bei der vorgelegten Monographie handelt es sich insgesamt um eine höchst kenntnisreiche, sehr fleißige und die politikwissenschaftliche Europa- sowie Parteienforschung bereichernde Studie. Vernachlässigbare Schwächen offenbart sie allenfalls dort, wo der Leser gern noch mehr an Erklärung anhand der vom Verfasser geführten Interviews gewünscht hätte. Letztere über den Status der „Kontrolle und Veranschaulichung des Quantitativen“ (S. 361) zu hieven, wäre zwar erstrebenswert; aber das ändert überhaupt nichts am Gesamturteil: eine einwandfreie Arbeit, die ein Phänomen auf exzellente Weise, methodisch versiert und gekonnt aufschließt, so dass sie für jeden an Rekrutierungsprozessen Interessierten uneingeschränkt empfohlen werden kann. 